TRANSACTIONS OF THE

AMERICAN MATHEMATICAL SOCIETY

Volume 191, 1974

\title{
GENERALIZED ALMOST PERIODICITY IN GROUPS $\left({ }^{1}\right)$
}

BY

\author{
HENRY W. DAVIS
}

\begin{abstract}
A module of almost periodic functions on a group is closed with respect to a quite general seminorm. The new space of functions is characterized in terms of the internal properties of its members. This yields new characterizations of Besicovitch and Weyl almost periodic functions in a variety of group-theoretic settings. Eberlein's theorem that weakly almost periodic functions on the real line are Weyl almost periodic is extended to locally compact groups.
\end{abstract}

1. Introduction. Let $\alpha(G)$ be a module of von Neumann AP (=almost periodic) functions on a group $G$. Using a transformation $L$ we define a seminorm \| \| with which we close $\alpha(G)$, obtaining a larger space $\alpha_{L}(G)$ (see 2.2). Our procedure extends to groups the Besicovitch-Bohr procedure of closing the trigonometric polynomials on the real line [4, Chapter 2]. Due to the abstract way $L$ is defined, our results include a wide variety of Besicovitch and Weyl-like AP functions on groups (see 2.3).

The space $\left(\alpha_{L}(G),\|\|\right)$ is a seminormed linear space and $L$ is an invariant mean on $a_{L}(G)$. The main theorem of the paper (4.9) characterizes the functions $f \in a_{L}(G)$ in terms of their internal properties. We obtain two conditions: condition (A) is a rather standard almost periodicity condition (slightly disguised, see $4.10,4.11)$. Condition (B) is that $|f(x t)-f(x)| b(x)$ must satisfy a kind of weak Fubini theorem for certain $b \in a(G)$. When applied to the classical Besicovitch and Weyl AP functions on the real line our results are new (see 4.12).

In $\$ 5$ we give a Bohr-like expression for the mean value of weakly AP functions on locally compact topological groups. Using this we extend Eberlein's result that on the real line weakly AP functions are Weyl AP. In most nonabelian cases we only conclude that they are Besicovitch AP.

In $\$ 3$ we state some facts about modules which are used in $\$ 4$.

2. Notation, definitions and examples.

2.1 Notation. Let $G$ be a group. $e$ denotes the identity of $G$ and $A P(G)$ is the set of complex-valued von Neumann AP functions on $G$. $M f$ is the mean value of $f \in A P(G)$ and $f \times g$ is the convolution of $f, g \in A P(G)$. We shall say $\delta \subset A P(G)$

Received by the editors June 6, 1972 and, in revised form, June 25, 1973. AMS (MOS) subject classifications (1970). Primary 43A60; Secondary 42 A84.

(1) Work performed under the auspices of the U. S. Atomic Energy Commision. 
is an invariant vector space of $A P$ functions if $\mathcal{S} \neq \varnothing$ and

(i) $f, g \in \mathcal{S}, \alpha, \beta$ complex $\Rightarrow \alpha f+\beta g \in \mathcal{S}$,

(ii) $f=\mathcal{E}, x, y \in G \Rightarrow{ }_{x} f_{y} \in \mathcal{S}$, where we define ${ }_{x} f_{y}(t)=f(x t y)$.

Using the terminology of van Kampen [18], we say $\mathcal{S}$ is a module if in addition to (i), (ii) we have

(iii) $f, g \in \mathcal{S} \Rightarrow f g \in \mathcal{S}$ ( $f g$ denotes pointwise multiplication),

(iv) $f \in \mathcal{S} \Rightarrow \bar{f} \epsilon \mathcal{S}(\bar{f}=$ conjugate of $f)$,

(v) $\mathcal{S}$ is closed, i.e., $f_{n} \in \mathcal{S},\left\|f_{n}-f\right\|_{\infty} \underset{n}{\longrightarrow} 0 \Rightarrow f \in \mathcal{S}$.

Here $f$ is a complex-valued function on $G$ and \|\|$_{\infty}$ denotes the supremum norm. We say a module is nontrivial iff it contains a nonzero function.

If $(G, \mathcal{T})$ is a topological group, $A P(G, \mathfrak{T})$ is the set of $\mathcal{T}$-continuous members of $A P(G)$. If $(G, \mathcal{J})$ is a locally compact $T_{0}$ topological group (= LC group), then $\mu$ denotes left Haar measure on $G . L_{1, l o c}(G)$ is the set of $\mu$-measurable complexvalued functions $f$ on $G$ such that $\int_{E} f d \mu$ exists and is finite for all compact $E \subset G$.

Let $R, C$ denote, respectively, the set of real and complex numbers. If $\mathcal{F}$ is a set of complex-valued functions, $\mathcal{F}^{r}$ denotes its real-valued members. The symmetric differences of two sets $A, B$ is denoted $A \Delta B$. Let $f: G \rightarrow C$. We define

$$
\begin{aligned}
& E_{1}(\epsilon, f)=\left\{x \in G:\left\|_{x} f-f\right\|_{\infty}<\epsilon\right\}, \\
& E_{2}(\epsilon, f)=\left\{x \in G:\left\|f_{x}-f\right\|_{\infty}<\epsilon\right\} .
\end{aligned}
$$

$R f$ denotes the real part of $f$ and, if $f$ is real-valued, $f^{+}, f^{-}$denote its positive and negative parts. Finally, by an $\epsilon$-mes $b$ in a metric space is meant a finite set of points of the space such that each point of the space is within $\epsilon$ of some member of the finite set.

2.2 Definition. Let $\alpha(G)$ be a nontrivial module of AP functions on $G$. Let $\mathcal{E}$ be a set of complex-valued functions on $G$ satisfying

(E1) $f, g \in \mathcal{G}, \alpha, \beta \in C \Rightarrow \alpha f+\beta g \in \mathcal{E}$;

(E2) $f \in \mathcal{E}, x, y \in G \Rightarrow{ }_{x} f_{y} \in \mathcal{E}$;

(E3) $f \in \tilde{E} \Rightarrow \bar{f} \in \tilde{E}$;

(E4) $f \in \mathcal{E} \Rightarrow|f| \in \mathcal{E}$;

(ES) है $\supset \alpha(G)$;

(E6) $f \in \mathcal{G}, g \in \alpha(G) \Rightarrow f_{g} \in \mathcal{G}$.

Let $\mathscr{D}$ be a set of extended real-valued functions on $G$ and let $L: D \rightarrow$ $[-\infty, \infty]$. Assume

(D1) $\mathfrak{D} \supset \varepsilon^{r}$.

(D2) $f \in \mathfrak{D} \Rightarrow|f| \in \mathcal{D}$.

(D3) $f, g \in \mathfrak{D} \Rightarrow f+g \in \mathfrak{D}$.

(L1) $0 \leq f \in \mathcal{E}^{r}, x, y \in G \Rightarrow L\left({ }_{x} f_{y}\right)=L \%$

(L2) $\lambda \geq 0, \lambda f, f \in \mathscr{D} \Rightarrow L(\lambda f)=\lambda L f$. 
(L3) $|f|,-|f| \in \mathfrak{D} \Rightarrow-L(-|f|) \leq L|f|$.

(L4) $f, g \in \mathfrak{D}, f \leq g \Rightarrow L f \leq L_{g}(f \leq g$ means that $f(t) \leq g(t)$ for all $t \in G)$.

(L5) $f, g \in \mathfrak{D} \Rightarrow L(f+g) \leq L f+L g$ whenever the right side is well defined, i.e., is $\neq \infty-\infty$.

(L6) $f \in a\left(G^{r}\right) \Rightarrow L f=M f$.

(DL) For every $f \in \mathcal{E}, b \in a(G)^{r}$ one has $L_{x}|f(x t)-f(t)| b(x) \in \mathcal{D}$, as a function of $t$, provided that either $L|f|<\infty$ or $h \equiv 1$.

Here we use the notation $L_{x}(g(x))=L g$. Define $\|f\|=L|f|$ for all $f \in \mathcal{G}$. Given such a system $a(G), \delta, \mathscr{D}, L$ we then define $\alpha_{L}(G)$ to be the set of all $f \in \mathcal{E}$ such that for every $\epsilon>0$ there exists $g \in \alpha(G)$ satisfying $\|f-g\|<\epsilon$. We keep this definition of $a_{L}(G)$ even if $L$ fails to satisfy (DL) (cf. 2.3(g), (h)).

2.3 Examples. We use the fact that any set of the form $A P(G, \mathfrak{J})$-which includes $A P(G)$-is a nontrivial module. Actually these are the only nontrivial modules (3.3).

(a) Let $G=R$ be made a group under addition and let $R$ have the usual topology, $\mathcal{T}$. Let $a(R)=A P(R, \mathcal{T})$ be the Bohr AP functions.

Let

$$
\xi=L_{1, \mathrm{loc}}(G)
$$

(2) $\mathscr{D}=\left\{f: f\right.$ is a $\mu$-measurable function from $G$ to $(-\infty, \infty], f \geq g$ for some $\left.g \in \mathscr{E}^{r}\right\}$.

Define $L$ on $\mathscr{D}$ by

$$
L f=\varlimsup_{T \rightarrow \infty} \frac{1}{2 T} \int_{-T}^{T} f d \mu .
$$

(L1) is satisfied because for $0 \leq f \in \mathcal{E}^{r}$ we have

$$
L f_{a}=\varlimsup_{T \rightarrow \infty} \frac{1}{2 T} \int_{-T+a}^{T+a} f d \mu \leq \varlimsup_{T \rightarrow \infty} \frac{2(T+|a|)}{2 T} \frac{1}{2(T+|a|)} \int_{-(T+|a|)}^{T+|a|} f d \mu=L f,
$$

which includes the opposite inequality. To see that $(D L)$ is satisfied take $f \in \mathcal{G}$, $b \in a(R)^{r}$ and define $\phi(t)=L\left|f_{t}-f(t)\right| b . \phi$ is measurable because we need only consider rational $T$ in the definition of $L$. If $b \equiv 1, \phi \geq 0$ so $\phi \in \mathcal{D}$. If $L|f|<\infty$, then $\phi \in \mathscr{D}$ because

$$
\phi(t) \geq-\|b\|_{\infty}[L|f|+|f(t)|]
$$

The other conditions of 2.2 are easy to check. $\alpha_{L}(R)$ is the set of Besicovitch AP functions on $R$.

(b) Let $G, a(G), \mathcal{E}, \mathfrak{D}$ be as in (a). For $f \in \mathfrak{T}$ define

$$
L f=\lim _{T \rightarrow \infty} \sup _{x \in C} \frac{1}{T} \int_{-T}^{T} f_{x} d \mu .
$$

$L\left|f_{t}-f(t)\right| b$ is measurable in $t$ because we need only consider rational $T, x$ in the definition of $L . a_{L}(R)$ is the set of Weyl AP functions on $R$. 
(c) Let $(G, \mathcal{T})$ be an LC group of the form $R^{a} \times Z^{b} \times F$, where $Z=$ $\{0, \pm 1, \ldots\}, F$ is a compact group and $a, b \geq 0$ are integers. Such groups include the compactly generated abelian LC groups $[17,9.8]$. Let $a(G)=A P(G, \mathfrak{T})$ and let $\mathcal{E}, \mathcal{D}$ be given by (1), (2), above. Define $K_{n}$ on $\mathcal{D}$ by

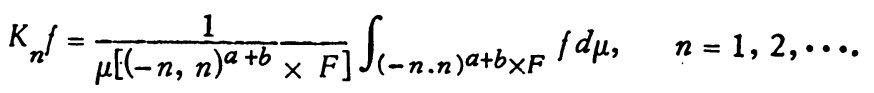

Define $L_{1}, L_{2}$ on $\mathcal{D}$ by

$$
L_{1} f=\varlimsup_{n \rightarrow \infty} K_{n} f, \quad L_{2} f=\lim _{n \rightarrow \infty} \sup _{x, y \in G} K_{n}\left(f_{x}\right) .
$$

$L_{2}\left|f_{t}-f(t)\right| b$ is a measurable function of $t$ because

$$
\sup _{x, y \in G} K_{n}\left[f_{x}\right]=\sup _{z \in R^{a} \times Z b} K_{n}\left[f_{(z, e)}\right]
$$

and one need only consider $z^{\prime}$ in the countable dense subset of $R^{a} \times Z^{b} \cdot a_{L_{1}}(G)$, $a_{L_{2}}(G)$ are, respectively, the Besicovitch and Weyl AP functions on $G$ (with respect to $\left.L_{1}, L_{2}\right)$.

(d) Let $(G, \mathscr{J})$ be a $\sigma$-compact abelian LC group. Let $\alpha(G)=A P(G, \mathcal{I})$ and let $\tilde{E}$ be the bounded members of $L_{1, \text { loc }}(G)$. Let $\mathcal{D}$ be given by (2). Let $\left\{V_{n}\right\}_{1}^{\infty}$ be a sequence of subsets of $G$ such that $0<\mu\left(V_{n}\right)<\infty$ for all $n$ and such that for each $x \in G$

$$
\lim _{n \rightarrow \infty} \frac{\mu\left(x V_{n} \Delta V_{n}\right)}{\mu\left(V_{n}\right)}=0 .
$$

Define $L$ on $\mathfrak{D}$ by

$$
L f=\varlimsup_{n \rightarrow \infty} \frac{1}{\mu\left(V_{n}\right)} \int_{V_{n}} f d \mu .
$$

For the fact that $\left\{V_{n}\right\}_{1}^{\infty}$ exists and that $(L G)$ is satisfied see $[17,18.10-18.14]$. (L1) follows because the members of $\xi$ are bounded (cf. proof of 18.10 in [17]). $\alpha_{L}(G)$ is the set of bounded Besicovitch AP functions on $G$, with respect to $L$. The general Besicovitch AP functions on $G$ are discussed in 4.14, below.

(e) Let $G$ be an arbitrary group and $a(G)=A P(G)$. Let $\delta$ be the set of all complex-valued functions on $G$ and let $D$ be the set of all functions from $G$ to $(-\infty, \infty]$. Set

$Q=\left\{\left(\left\{a_{r}\right\}_{r=1}^{n},\left\{a_{r}\right\}_{r=1}^{n}\right): a_{r} \in R, 0<\alpha_{r} \in G, \sum \alpha_{r}=1,1 \leq r \leq n ; n\right.$ is a positive integer $\}$.

Define $L$ on $D$ by

$$
L f=\inf \left\{\sup _{x, y \in G} \sum_{r} a_{r} f\left(x a_{r} y\right):\left(\left\{a_{r}\right\},\left\{a_{r}\right\}\right) \in \mathbb{G}\right\} .
$$

It is not hard to verify the conditions of 2.2. We call $a_{L}(G)$ the Følner-Weyl AP functions on G. They are discussed in [13] and [7]. 
(f) Let $G$ be an infinite group, $a(G)=A P(G)$ and $E, D$ as in (e), above. There exists a pairwise disjoint sequence $E_{1}, E_{2}, \ldots$ of symmetric subsets of $G$ with the property that for every finite set $\left\{a_{1}, \ldots, a_{n}\right\} \subset G$

$$
\bigcap_{1 \leq i, i \leq n} a_{i} E_{b} a_{j} \neq \varnothing, \quad b=1,2, \ldots
$$

Let $Q$ be as in (e). For $b=1,2 \ldots$ and $f \in T$ define

$$
\bar{M}_{b} f=\inf _{A, B, D} \sup _{x, y} \sum_{i=1}^{n} a_{i} f\left(x a_{i} y\right),
$$

where $A=\left(\left\{a_{i}\right\},\left\{a_{i}\right\}\right) \in \mathbb{Q}, B=\left\{b_{1}, \cdots, b_{k}\right\} \subset G, D=\left\{d_{1}, \ldots, d_{k}\right\} \subset G$, and the supremum is to be taken over the nonempty set of those $x, y \in G$ for which $b_{j} x a_{i} y d_{j} \in E_{b}$ whenever $1 \leq j \leq k, 1 \leq i \leq m$. Now define $L$ on $D$ by $L /=$ $\lim _{b \rightarrow \infty} \vec{M}_{b} f$. For the fact that the sequence $E_{b}$ exists and that $L$ satisfies the conditions of 2.2 see [14]. We call $a_{L}(G)$ the Folner-Besicovitch AP functions on $G$. $\left(a_{L}(G),\|\|\right)$ is complete and has properties very analogous to the usual Besicovitch AP functions considered in (a). See [14].

(g) Let $(G, \mathcal{J})$ be an abelian LC group. Let $a(G)=A P(G, \mathcal{I})$ and let $\tilde{E}$ be the bounded members of $L_{1,10 c}(G)$. Let $D$ be given by (2). Since $G$ is amenable, there is a net $\left(V_{d}, d \in D, \geq\right)$ of subsets of $G$ such that $0<\mu\left(V_{d}\right)<\infty$ for all $d$ and such that for all $x \in G$

$$
\lim _{d \in D} \frac{\mu\left(x V_{d} \Delta V_{d}\right)}{\mu\left(V_{d}\right)}=0
$$

(cf. [15, p. 43] and [11]). Define $L$ on $D$ by

$$
L f=\varlimsup_{d \in D} \frac{1}{\mu\left(V_{d}\right)} \int_{V_{d}} f d \mu
$$

The conditions of 2.2 are satisfied except possibly the requirement in (DL) that $L\left|f_{t}-f(t)\right| b$ be a measurable function of $t$. We return to this problem in 4.13. $a_{L}(G)$ is the set of bounded Besicovitch AP functions on $G$, with respect to $L$. The general Besicovitch AP functions on $G$ are discussed in 4.14.

(h) Let $(G, \mathcal{I})$ be an $L C$ group. Let $\alpha(G)=A P(G, \mathcal{I})$ and let $\mathcal{E}, \mathcal{D}$ be given by (1), (2). Let $\left(V_{d}, d \in D, \geq\right)$ be a net of subsets of $G$ such that $0<\mu\left(V_{d}\right)<\infty$ for every $d$ and such that for every $f \in a(G)$

$$
\lim _{d \in D} \frac{1}{\mu\left(V_{d}\right)} \int_{V_{d}} f d \mu=M f \text {. }
$$

Such a net exists by 3.4 of [6]. Define $L$ on $D$ by

$$
L f=\varlimsup_{d \in D} \sup _{x, y \in G} \frac{1}{\mu\left(V_{d}\right)} \int_{V_{d} x^{f}} d \mu .
$$

Again the conditions of 2.2 are satisfied except possibly the measurability in $t$ 
of $L\left|f_{t}-f(t)\right| b$. We consider this problem in 4.13. $a_{L}(G)$ is the set of Weyl AP functions on $G$, with respect to $L$.

3. Preliminaries on modules. Let $\left\{D^{\lambda}: \lambda \in \Lambda\right\}$ be a complete(2) set of inequivalent irreducible unitary finite-dimensional representations of the group $G$. We shall let $s_{\lambda}$ denote the degree of $D^{\lambda}$ and let $\mathbb{M}^{\lambda}$ be the finite-dimensional subspace of $A P(G)$ spanned by $\left\{D_{\rho \sigma}^{\lambda}\right\}_{\rho, \sigma=1}^{s_{\lambda}}$. For $\Lambda_{0} \subset \Lambda$ define $\Sigma_{\lambda \in \Lambda_{0}} M^{\lambda}$ to be smallest closed vector subspace of $A P(G)$ containing $\pi^{\lambda}$ for every $\lambda \in \Lambda_{0}$. Take $f \in A P(G)$. Maak $[19$, p. 141] defines the summation module $[f]$ determined by $f$ by $[f]=\Sigma_{k=1}^{\infty} \pi^{\lambda_{k}}$, where the $\lambda_{k}$ are indices of those representations occurring nontrivially in the Fourier series of a certain sequence of weight functions. We shall denote $\left\{\lambda_{k}\right\}_{1}^{\infty}$ by $\Lambda(f)$.

Let $g: G \rightarrow C$. We say $\left\{A_{1}, \ldots, A_{n}\right\}$ is an $\epsilon$-covering of $G$ with respect to $g$ iff

$$
G=\bigcup_{i=1}^{n} A_{i} \text { and } \sup _{a, b \in G}|g(a x b)-g(a y b)|<\epsilon
$$

whenever $x, y \in A_{i}$ for some $i$. One can show that $g \in A P(G)$ iff for every $\epsilon>0$ $G$ has an $\epsilon$-covering with respect to $g$ (this is the definition of $A P(G)$ in [19]). Let $f, g \in A P(G)$. We say $g$ is just as $A P$ as $f$ iff for every $\epsilon>0$ there exists $\delta>0$ such that every $\delta$-covering of $G$ with respect to $f$ is an $\epsilon$-covering of $G$ with respect to $g$. The following theorem (3.2) gives alternative descriptions of [f]. (i) is due to Maak [19, p. 143].

3.1 Lemma. Let $f \in A P(G)$. There exists a smallest topology $\mathscr{T}(f)$ for $G$ sucb that $(G, \mathcal{T}(f))$ is a topological group and $f$ is $\mathscr{T}(f)$-continuous. The sets

$$
V(\epsilon, f)=\left\{x \in G: \sup _{a, b \in G}|f(a x b)-f(a b)|<\epsilon\right\}, \quad \epsilon>0,
$$

are a fundamental neigbborbood system of $e$ in $\mathscr{T}(f)$.

3.2 Theorem. Let $f \in A P(G)$.

(i) [f] consists of those AP functions on $G$ which are just as $A P$ as 1.

(ii) $[f]=A P(G, \mathcal{T}(f))$.

Proof of 3.1. Notice that $e \in V(\epsilon, f)^{-1}=V(\epsilon, f), V(\epsilon, f)^{2} \subset V(2 \epsilon, f)$ and $y V(\epsilon, f) y^{-1}=V(\epsilon, f)$ for all $y \in G$. Thus $\{V(\epsilon, f)\}_{\epsilon>0}$ is a basic neighborhood system of $e$ in a topology $\mathscr{T}(f)$ such that $(G, \mathcal{T}(f))$ is a topological group. If $x_{n} \rightarrow x(\mathcal{T}(f))$, then $x_{n} x^{-1}$ is eventually in every $V(\epsilon, f)$ from which it follows that $\left|f\left(x_{n}\right)-f(x)\right|$ is eventually $<\epsilon$ for every $\epsilon>0$. Hence $f$ is $\mathcal{T}(f)$-continuous.

To complete the proof we must show that if $f \in A P(G, \mathfrak{T})$ then $\mathfrak{T} \supset \mathfrak{T}(f)$. To

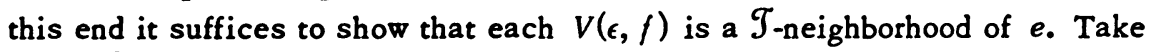

(2) I.e., every irreducible (finite-dimensional) representation of $G$ is equivalent to some $D^{\lambda}[19, \S 30]$. 
$\epsilon>0$ and let $\left\{a_{i} f_{b_{i}}\right\}_{i=1}^{n}$ be an $\epsilon$-mesh in $\left(\left\{_{a} f_{b}: a, b \in G\right\},\|\|_{\infty}\right)$. Let $U$ be a $\mathfrak{T}$ neighborhood of $e$ such that

$$
\left|a_{i} f_{b_{i}}(x)-a_{i} f_{b_{i}}(e)\right|<\epsilon \text { for all } x \in U, 1 \leq i \leq n \text {. }
$$

Now take any $x \in U$ and any $a, b \in G$. Take $i \in\{1, \cdots, n\}$ such that $\left\|a_{i} f_{b_{i}}-{ }_{a} f_{b}\right\|_{\infty}<\epsilon$. Then

$|f(a \times b)-f(a b)| \leq\left|f(a x b)-f\left(a_{i} x b_{i}\right)\right|+\left|f\left(a_{i} x b_{i}\right)-f\left(a_{i} b_{i}\right)\right|+\left|f\left(a_{i} b_{i}\right)-f(a b)\right|<3 \epsilon_{0}$.

As $x \in U, a, b \in G$ are arbitrary, $U \subset V(3 \epsilon, f)$. As $\epsilon>0$ is arbitrary and $U$ is a

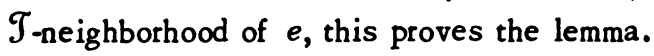

Proof of 3.2. We use 3.1 and (i) to prove (ii). $g \in[f]$ iff [for every $\epsilon>0$ there exists $\delta>0$ such that $\sup _{a, b \in G}|f(a u b)-f(a v b)|<\delta$ implies $\left.\sup _{a, b \in G}|g(a u b)-g(a v b)|<\epsilon\right]$ iff [for every $\epsilon>0$ there exists $\delta>0$ such that for every $v \in G \nu V(\delta, f) \subset v V(\epsilon, g)]$ iff $[\mathcal{T}(f) \supset \mathcal{T}(g)]$ iff $g \in A P(G, \mathcal{T}(f))$.

3.3 Theorem. Let $\alpha(G)$ be an invariant vector space of AP functions on $G$. The following are equivalent:

(i) $\alpha(G)$ is a nontrivial module;

(ii) $\alpha(G)=A P(G, \mathfrak{J})$ for some topology $\mathfrak{T}$;

(iii) $\alpha(G)$ is closed and $f \in \alpha(G)$ implies [ $f] \subset \alpha(G)$.

Proof. That (i) implies (ii) is known. Since we do not know where an explicit proof may be found, we outline a proof here: Let $A=\alpha(G)$ and let $T_{x}$ : $A \rightarrow A$ by $T_{x} f={ }_{x-1} f$ for all $x \in G$. Let $A$ have the topology induced by \|\|$_{\infty}$. Let $\bar{G}$ be the closure in $A^{A}$ of $T(G)$ and let $\mathcal{P}$ be the restriction to $\bar{G}$ of the product topology for $A^{A}$. ( $\left.\bar{G}, \mathcal{P}\right)$ is a compact $T_{2}$-topological group under composition and $T: G \rightarrow \bar{G}$ is a homomorphism with ker $T=\{x \in G: f(x)=f(e)$ for all $f \in A\}$. The set of finite intersections of $\{V(\epsilon, f): f \epsilon A, \epsilon>0\}$ is a local neighborhood base at $e$ for a topology $\mathcal{J}$ such that $(G, \mathcal{J})$ is a topological group and $A \subset A P(G, \mathcal{I}) . T$ is $\mathfrak{T}$-continuous and the map of $(G, \mathfrak{I}) \rightarrow(T(G), \mathcal{P} / T(G))$ by $x \rightarrow T_{x}$ is open. For each $f \in A$ define $\bar{f}: \bar{G} \rightarrow C$ by $\bar{f}(R)=R^{-1}(f)(e)$ for all $R \in \bar{G}$. Then $\bar{f}\left(T_{x}\right)=f(x)$ when $x \in G$; also $\bar{f} \in C(\bar{G})$, the $\mathcal{P}$-continuous functions on $\bar{G}$. Letting $\bar{A}=\{\bar{f}: f \in A\}$ one can show by Stone-Weierstrass that $\bar{A}=$ $C(\bar{G})$. Finally if $b \in A P(G, \mathfrak{J}), b$ is constant on the cosets of $\operatorname{ker} T$ so we may define $H$ on $T(G)$ by $H\left(T_{x}\right)=b(x)$. Since $x \rightarrow T_{x}$ is open, $H \in A P(T(G), \mathcal{P} / T(G)$ ). Let $\bar{H} \in A P(\bar{G}, \mathscr{P})=C(\bar{G})$ extend $H . \bar{H}$ exists by direct proof or by [2]. But then there exists $f \in A$ such that $\bar{f}=\bar{H}$ from which it follows that $b=f \in A$. Hence $A P(G, \mathcal{J}) \subset A$, completing the proof of (ii).

That (ii) implies (iii) follows from 3.2(ii).

Assume (iii) holds. Since $[0]=$ the constant functions, $a(G)$ is nontrivial. If $f \in a(G)$, then $\bar{f} \in[f] \subset a(G)$. Thus to prove (i) we need only show that if 
f. $g \in a(G)$, then $f g \in a(G)$. But $f^{2} \in[f], g^{2} \in[g],(f+g)^{2} \in[f+g]$, by 3.2 , so that by combining these we get $f g \in a(G)$. This proves the theorem.

3.4 Remarks. (1) Let $\alpha(G)$ be a nontrivial module. We use in the sequel the fact that $a(G)$ has the following closure properties (which follow easily from the above): $\alpha(G)$ contains the constant functions; $f \in \alpha(G)$ implies that $|f|, R /$, $(R /)^{ \pm} \in \alpha(G) ; f, g \in a(G)$ implies that $f \times g, M\left|f_{t}-f(t)\right|_{g} \in \alpha(G)$, the latter as a function of $t$.

(2) Every closed invariant vector space of AP functions on $G$ may be written in the form

$$
a(G)=\sum_{m^{\lambda} \subset a(G)} \pi^{\lambda}
$$

([19, p. 132]; Maak uses the term "module" differently than we). If $G$ is abelian, each $\pi^{\lambda}$ is the one-dimensional subspace spanned by some $\lambda \in G^{*}$, the dual group of $G$. In this case the nontrivial modules on $G$ are precisely the sets $\Sigma_{\lambda \in X^{*}} \pi^{\lambda}$, where $X^{*}$ runs through the subgroups of $G^{*}$ (cf. [1]). For example, let $\mathcal{T}$ be the usual topology for $R$ and let $a(R)=A P(R, \mathfrak{I})$ be the Bohr AP functions. If $0 \not b \in a(R)$, then $\Lambda(b)$ is the additive subgroup of $R$ generated by the Fourier exponents of $b$; if $b \equiv 0$, then $\Lambda(b)=\{0\}$.

The following two lemmas will be used in $\$ 4$. Recall the definition of $E_{1}$, $E_{2}$ in $\$ 2.1$.

3.5 Lemma. Let $g: G \rightarrow C, f \in A P(G)$. Take $j \in\{1,2\}$. Suppose that for every $\epsilon>0$ there exists $\delta>0$ sucb that $E_{j}(\delta, f) \subset E_{j}(\epsilon, g)$. Then $g \in[f]$.

Proof. Take, for example, $j=2$. For any $b: G \rightarrow C$ and $\eta>0$ notice that $\left\{b_{a_{i}}\right\}_{i=1}^{n}$ is an $\eta$-mesh in $\left(\left\{b_{a}: a \in G\right\},\|\|_{\infty}\right)$ iff $G=\bigcup_{i=1}^{n} a_{i} E_{2}(\eta, b)$. It follows that $g \in A P(G)$. To show that $g \in[f]$ it suffices to show that $g$ is $\mathcal{T}(f)$-continuous. To this end it suffices to show that for any $b \in A P(G)$ the finite intersections of sets in $\left\{a E_{2}(b, \epsilon) a^{-1}: a \in G, \epsilon>0\right\}$ are a base for the $\mathcal{T}(b)$-neighborhood system of $e$. Since $V(\epsilon, b) \subset E_{2}(\epsilon, b)$ for all $\epsilon>0$ and $a V(\epsilon, b) a^{-1}=V(\epsilon, b)$, it suffices to show that each $V(\epsilon, b)$ contains an appropriate finite intersection.

Take $\epsilon>0$. Let $\left\{b_{a_{i}}\right\}_{i=1}^{n}$ be an $\epsilon$-mesh in $\left(\left\{b_{a}: a \in G\right\},\|\|_{\infty}\right)$. Take any $x \in \bigcap_{i=1}^{n} a_{i} E_{2}(\epsilon, b) a_{i}^{-1}$. Take arbitrary $a, b \in G$. Take $i_{0}$ such that $b \epsilon$ $a_{i_{0}} E_{2}(\epsilon, b)$. Then

$$
|b(a x b)-b(a b)| \leq\left|b(a x b)-b\left(a x a_{i 0}\right)\right|+\left|b\left(a x a_{i 0}\right)-b\left(a a_{i 0}\right)\right|+\left|b\left(a a_{i 0}\right)-b(a b)\right| .
$$

The first and third terms above are $\leq\left\|b_{b}-b_{a_{i}}\right\|_{\infty}<\epsilon$, since $b \in a_{i_{0}} E_{2}(\epsilon, b)=$ $\left\{y \in G:\left\|b_{y}-b_{a_{i 0}}\right\|_{\infty}<\epsilon\right\}$. As $e, x \in a_{i_{0}} E_{2}(\epsilon, b) a_{i_{0}}^{-1}$, the second term is of the form $\left|b(a c)-b\left(a c^{\prime}\right)\right|$, where $c, c^{\prime} \in a_{i_{0}} E_{2}(\epsilon, b)$. Hence $|b(a \times b)-b(a b)|<4 \epsilon$. It follows that $\bigcap_{i=1}^{n} a_{i} E_{2}(\epsilon, b) a_{i}^{-1} \subset V(4 \epsilon, b)$, proving the lemma. 
3.6 Lemma. Let $\Phi \in A P(G)$. There exists a sequence of weight functions $b_{n} \in[\Phi]$ sucb that $\left\|g \times b_{n}-g\right\|_{\infty} \underset{n}{\longrightarrow} 0$ for all $g \in[\Phi]$. We may require that

$$
b_{n}(x)=\sum_{\nu \in \Lambda(\Phi)} s^{\nu} \beta_{n}^{\nu} \sum_{\rho=1}^{s^{\nu}} D_{\rho \rho}^{\nu}(x), \quad n=1,2, \cdots,
$$

where the right side converges uniformly and each $\beta_{n}^{\nu} \geq 0$. (By saying $b_{n}$ is a wergint function is meant that $M b_{n}=1$ and $b_{n} \geq 0, n=1,2, \ldots$ )

Proof. Let $g_{n}$ be the weight functions considered in [19, pp. 139-143, Theorems 3 through 6]. Define $b_{n}=g_{n} \times g_{n}, n=1,2, \ldots$. Evidently each $b_{n}$ is a weight function and since

$$
g_{n} \sim \sum_{\nu \in \Lambda(\Phi)} s^{\nu} \gamma_{n}^{\nu} \sum_{\rho=1}^{\nu} D_{\rho \rho}^{\nu}
$$

the last assertion follows with $\beta_{n}^{\nu}=\left|\gamma_{n}^{\nu}\right|^{2}$.

From Theorem 4, p. 140 of [19] and the definition of $\Lambda(\Phi)$, we have $\lim _{n \rightarrow \infty} \beta_{n}^{\nu}=1$ for all $\nu \in \Lambda(\Phi)$. For each $g \in[\Phi], g \times b_{n}$ is majorized by $g$ and bounded by $\|g\|_{\infty}$. Hence by Theorem 1, p. 136 of [19], $\left\|g \times b_{n}-g\right\|_{\infty} \underset{n}{\longrightarrow} 0$. Finally $b_{n}=g_{n} \times g_{n} \in[\Phi]$ by $3.4(1)$ since $g_{n} \in[\Phi]$. This proves the lemma.

3.7 Remark. We shall use the fact that, each $b_{n}$ being real-valued,

$$
b_{n}=\sum_{\nu \in \Lambda(\phi)} s^{\nu} \beta_{n}^{\nu} \sum_{\rho=1} R D_{\rho \rho}^{\nu}, \quad n=1,2, \ldots
$$

4. Characterization of $a_{L}(G)$. We continue the notation of $\$ \$ 2$ and 3 . Unless stated otherwise we assume that $a(G), \mathcal{G}, \mathfrak{D}$, and $L$ satisfy all the conditions (E1), (D1), (L1) and (DL) of 2.2: For $f \in \mathcal{E}$ define $\Delta_{f}, \Delta_{f}^{r}$ on $G$ by $\Delta_{f}(x)=$ $\left\|_{x} f-f\right\|, \Delta_{f}^{r}(x)=\left\|f_{x}-f\right\|$ for all $x \in G$. For $\epsilon>0, f \in \mathcal{E}$ define

$$
L E_{1}(\epsilon, f)=\left\{x \in G: \Delta_{f}(x)<\epsilon\right\}, \quad L E_{2}(\epsilon, f)=\left\{x \in G: \Delta_{f}^{r}(x)<\epsilon\right\} .
$$

In the sequel we shall use freely the conditions (E1), (D1), (L1) and (DL) of 2.2 without always stating them explicitly.

4.1 Lemma. Let $f \in \mathcal{E}$ and $\Delta, \in a(G)$. Then both sides of

$$
L_{t} L_{x}|f(x t)-f(t)| \leq L_{x} L_{t}|f(x t)-f(t)|
$$

are well defined. If (*) bolds, then $L|f|<\infty$.

Proof. The left side of $(*)$ is well defined by $(\mathrm{DL})$ and the right side is simply $M \Delta_{f}$. Assume $(*)$ is true. Now $L|f| \leq L_{x}|f(x t)-f(t)|+|f(t)|$ by (L1), (L4), (L5) and (LG). If $L|f|=\infty$, then since $|f(t)|<\infty$ for all $t$, we have $L_{x}|f(x t)-f(t)|=\infty$ for all $t$. By $(*)$ 


$$
\infty=L_{t} L_{x}|f(x t)-f(t)| \leq M \Delta_{f},
$$

a contradiction. Therefore $L|f|<\infty$.

4.2 Remark. Let $f \in \mathcal{E}$ and $\Delta_{f} \in a(G)$. Let us show that the following condition is well defined:

(B) $L_{t} L_{x}|f(x t)-f(t)| R_{\rho \rho}^{\lambda}(x) \leq L_{x} L_{t}|f(x t)-f(t)| R_{D}^{\lambda}{ }_{\rho \rho}^{\lambda}(x)$ for all $\lambda \in \Lambda\left(\Delta_{f}\right)$ and $1 \leq \rho \leq s_{\lambda}$.

Taking $\lambda_{0} \in \Lambda$ such that $D^{\lambda_{0}} \equiv 1$, notice that $\lambda_{0} \in \Lambda\left(\Delta_{f}\right)$ (for example, by 3.2; or the definition of $\left.\left[\Delta_{f}\right]\right)$. Thus the inequalities of $(B)$ include $(*)$ of 4.1, which by 4.1 is well defined. From the truth of (*) we get that $L|f|<\infty$. Notice that $D_{\rho \rho}^{\lambda} \in \alpha(G)$ when $\lambda \in \Lambda(f)$ by 3.3. Thus by (DL) the left sides of the other inequalities in (B) are well defined. The right side of (B) is simply $M\left(\Delta_{f} R D_{\rho \rho}^{\lambda}\right)$.

4.3 Lemma. Suppose $f \in \mathcal{E}, \Delta_{f} \in \alpha(G)$. Set $\Phi=\Delta_{f}$ in 3.6 and let $b_{n}$ be as in that lemma. If (B) bolds, then

$$
L_{t} L_{x}|f(x t)-f(t)| b_{n}(x) \leq L_{x} L_{t}|f(x t)-f(t)| b_{n}(x), \quad n=1,2, \ldots
$$

Proof. The right side of $(1)$ is $M\left(\Delta_{f} b_{n}\right)$ and the left side is well defined by (B), (4.1) and (DL). If $\Delta_{f} \equiv 0$, it turns out that the $g_{n}$ of 3.6 are identically 1 , whence $b_{n} \equiv 1$ and (1) is a restatement of (B). Thus we may suppose $\Delta_{f} \not 0$.

Fix $n$ arbitrarily and set $K=b_{n}$. Take $\epsilon>0$ and set

$$
\delta=\epsilon / M \Delta
$$

By 3.7

$$
\|K-P\|_{\infty}<\delta
$$

where

$$
P=\Sigma^{*} \beta_{\rho}^{\lambda} R_{\rho \rho}^{\lambda}, \quad \beta_{\rho}^{\lambda} \geq 0,
$$

and $\Sigma^{*}$ denotes a finite sum. If $b \in a(G)$ is arbitrary,

$$
\begin{aligned}
L_{x}|f(x t)-f(t)| R b(x) & \geq L_{x}\left[-|f(x t)-f(t)|\|b\|_{\infty}\right] \text { by (L4) } \\
& \geq-\|b\|_{\infty} L_{x}|f(x t)-f(t)| \text { by (L2), (L3) } \\
& \geq-\|b\|_{\infty}[L|f|+|f(t)|] .
\end{aligned}
$$

Hence

$$
L_{t} L_{x}|f(x t)-f(t)| R b(x) \geq-2\|b\|_{\infty} L|f|>-\infty .
$$

This observation allows us to apply (L5) in the following chain of inequalities. 


$$
\begin{aligned}
& L_{t} L_{x}|f(x t)-f(t)| K(x) \\
& \quad \leq L_{t} L_{x}|f(x t)-f(t)|\left[\sum^{*} \beta_{\rho}^{\lambda} R D_{\rho \rho}^{\lambda}(x)+\delta\right] \text { by (3), (4), (L4) } \\
& \leq \sum^{*} \beta_{\rho}^{\lambda} L_{t} L_{x}|f(x t)-f(x)| R D_{\rho \rho}^{\lambda}(x)+\delta L_{t} L_{x}|f(x t)-f(t)| \\
& \quad \leq \sum^{*} \beta_{\rho}^{\lambda} L_{x} L_{t}|f(x t)-f(t)| R D_{\rho \rho}^{\lambda}(x)+\delta L_{x} L_{t}|f(x t)-f(t)| \quad \text { by (B) }(\text { B }),(L 2) \text { since } \beta_{\rho}^{\lambda} \geq 0 \\
& =M\left(\Delta_{f} P\right)+\delta M \Delta_{f} \\
& \leq M\left[\Delta_{f}(K+\delta)\right]+\delta M \Delta_{f} \text { by (3) } \\
& =M\left(\Delta_{f} K\right)+2 \delta M \Delta_{f} \\
& <L_{x} L_{t}|f(x t)-f(t)| K(x)+2 \epsilon \text { by (2). }
\end{aligned}
$$

As $\epsilon>0$ is arbitrary, (1) follows, proving the lemma.

4.4 Lemma. Take $f \in \mathcal{E}$ and $\epsilon>0$. If $\Delta_{f}^{r}$ is finite, then $E_{2}\left(\epsilon, \Delta_{f}^{r}\right)=$ $L E_{2}(\epsilon, f)$; if $\Delta_{f}$ is finite, $E_{1}\left(\epsilon, \Delta_{f}\right)=L E_{1}(\epsilon, f)$.

Proof. We consider $\Delta_{f}^{r}, \Delta_{f}$ being similar. Take $u \in G$.

$$
\begin{aligned}
\left\|\left(\Delta_{f}^{r}\right)_{u}-\Delta_{f}^{r}\right\|_{\infty} & =\sup _{x \in G}\left|\left\|f_{x u}-f\right\|-\left\|f-f_{x}\right\|\right| \\
& \leq \sup _{x \in G}\left\|f_{x u}-f_{x}\right\| \text { by (L5) since } \Delta_{f}^{r} \text { is finite } \\
& =\left\|f_{u}-f\right\| .
\end{aligned}
$$

Thus $L E_{2}(\epsilon, f) \subset E_{2}\left(\epsilon, \Delta_{f}^{r}\right)$. If $u \in E_{2}\left(\epsilon, \Delta_{f}^{r}\right)$, then

$$
\sup _{x \in G}\left|\left\|f_{x u}-f\right\|-\left\|f_{x}-f\right\|\right|<\epsilon .
$$

Taking $x=e$ gives $\left\|f_{u}-f\right\|<\epsilon$, whence $u \in L E_{2}(\epsilon, f)$. Therefore $E_{2}\left(\epsilon, \Delta_{f}^{r}\right) \subset$ $L E_{2}(\epsilon, f)$, proving the lemma.

4.5 Theorem. Let $f \in \mathcal{E}$. Suppose

$$
\Delta_{f}, \Delta_{f}^{r} \in \alpha(G)
$$

and that $f$ satisfies (B) of 4.2. Then $f \in \alpha_{L}(G)$.

$$
M\left(\Delta_{f} b_{n}\right)=M_{x}\left[\Delta_{f}(x) b_{n}\left(x^{-1}\right)\right]=\Delta_{f} \times b_{n}(e) \rightarrow \Delta_{f}(e)=0
$$

by 3.6. Take $n$ so large that the left side, above, is $\left\langle\epsilon / 2\right.$ and set $K=b_{n}$. Applying $3.3,3.6,4.3$ and (A) gives 


$$
\left\{\begin{array}{l}
K \in a(G), K \geq 0, \\
M K=1, M(\Delta, K)<\epsilon / 2, \\
(B) \text { holds with } R D_{\rho \rho}^{\lambda} \text { replaced by } K .
\end{array}\right.
$$

Write $f=u+i v$ where $u, v: G \rightarrow R$. By (E1), (E2), (E3), (EG) and (1) $u_{t} K$, $v_{t} K \in \mathcal{E}^{r}$ for all $t \in G$. Define $\Phi_{1}(t)=L\left(u_{t} K\right), \Phi_{2}(t)=L\left(v_{t} K\right)$ for all $t \in G$. The following string of inequalities shows that

$$
-\infty<\Phi_{1}(t)<\infty \text { for all } t \in G \text {. }
$$

They are justified by applying (L1), (L2), (L3), (L4) and recalling that by 4.1 and (B) $L|f|<\infty$.

$$
\begin{aligned}
-\infty & <-\|K\|_{\infty} L|f| \leq-\|K\|_{\infty} L|u|=-L_{x}|u(x t)|\|K\|_{\infty} \leq-L_{x}|u(x t)| K(x) \\
& \leq L_{x}[-|u(x t)| K(x)] \leq L\left(u_{t} K\right)=\Phi_{1}(t) \leq L\left|u_{t}\right| K \leq\|K\|_{\infty} L|u| \leq\|K\|_{\infty} L|f|<\infty .
\end{aligned}
$$

Similarly $\Phi_{2}$ is finite. (2) allows us to apply (LS) and (L4) to get

$$
\left|\Phi_{1}(t x)-\Phi_{1}(t)\right| \leq L\left|u_{t x}-u_{t}\right| K \leq\|K\|_{\infty}\left\|f_{x}-f\right\| \text { by (L1), (L2), (L4). }
$$

Consequently, by Lẹmma 4.4, $E_{2}\left(\rho /\|K\|_{\infty}, \Delta_{f}^{r}\right)=L E_{2}\left(\rho /\|K\|_{\infty}, f\right) \subset E_{2}\left(\rho, \Phi_{1}\right)$ for all $\rho>0$. By 3.5, $\Phi_{1} \in\left[\Delta_{f}^{r}\right] \subset \alpha(G)$. Similarly $\Phi_{2} \in a(G)$. Set $\Phi=\Phi_{1}+i \Phi_{2}$ so that $\Phi \in a(G)$.

Since $u(t)$ is finite for each $t \in G$, (L4), (L5) and (1) give $\left|L\left(u_{t} K\right)-u(t)\right| \leq$ $L\left|u_{t}-u(t)\right| K$, that is, $\left|\Phi_{1}(t)-u(t)\right| \leq L\left|u_{t}-u(t)\right| K$. Similarly for $\Phi_{2}$. Consequently

$$
|\Phi(t)-f(t)| \leq L\left|u_{t}-u(t)\right| K+L\left|v_{t}-v(t)\right| K \leq 2 L\left|f_{t}-f(t)\right| K,
$$

by (L4). Therefore, by (1), (L2) and (L4)

$$
\begin{aligned}
\|\Phi-f\| & =L_{t}|\Phi(t)-f(t)| \leq 2 L_{t} L_{x}|f(x t)-f(t)| K(x) \\
& \leq 2 L_{x} L_{t}|f(x t)-f(t)| K(x)=2 M\left(\Delta_{f} K\right)<\epsilon .
\end{aligned}
$$

Since $\Phi \epsilon \alpha(G)$ and $\epsilon>0$ is arbitrary, this proves the theorem.

4.6 Lemma. If $f \in a_{L}(G)$, then $L|f|<\infty$ and $\Delta_{f}, \Delta_{f}^{r} \in a(G)$.

Proof. Take $\epsilon>0$ and $g \in a(G)$ such that $\|f-g\|<\epsilon / 2$. Since $L|f|=\|f\| \leq$ $\|f-g\|+\|g\| \leq \epsilon / 2+\|g\|_{\infty}<\infty$, the first assertion is clear. To see that $\Delta_{f} \epsilon a(G)$ define $G(u)=\left\|_{u} g-g\right\|$. Then

$$
\left\|_{t} G-G\right\|_{\infty}=\sup _{x \in G}\left|\left\|_{t x} g-g\right\|-\left\|_{x} g-g\right\|\right| \leq\left\|_{t} g-g\right\| \leq\left\|_{t} g-g\right\|_{\infty} \text {. }
$$

Hence $E_{1}(\eta, g) \subset E_{1}(\eta, G)$ for all $\eta>0$. It follows from 3.5 that $G \in[g] \subset a(G)$. Also $\left\|_{u} f-f\right\| \leq\left\|_{u} f-{ }_{u} g\right\|+\left\|_{u} g-g\right\|+\|g-f\|$ and similarly with $f, g$ interchanged. This gives $\left|\Delta_{f}(u)-G(u)\right| \leq 2\|g-f\|<\epsilon$. Thus $\left\|\Delta_{f}-G\right\|_{\infty} \leq \epsilon$. As $\epsilon>0$ is arbitrary 
and $\alpha(G)$ is closed, $\Delta_{f} \in \alpha(G)$. Similarly $\Delta_{f}^{r} \in \alpha(G)$. Q.E.D.

4.7 Lemma. If $0 \leq f, f,-f \in \mathscr{L}$ and if $f$ is \|\| -approximatable by members of $a(G)^{r}$, then $L f<\infty$ and $L(-f)=-L /$.

Proof. Take $g_{n} \in a(G)^{r}$ such that $\left\|f-g_{n}\right\| \underset{n}{\rightarrow} 0$. If $\left\|f-g_{m}\right\|<1$, then $L f=\|f\| \leq\left\|f-g_{m}\right\|+\left\|g_{m}\right\| \leq 1+\left\|g_{m}\right\|_{\infty}<\infty$, proving the first assertion. Noting that $-\infty<-L f \leq L(-f) \leq 0$, we get from (L4), (L5)

$$
L\left(-g_{n}\right) \leq L\left|\left(-g_{n}\right)-(-f)\right|+L(-f) .
$$

Interchanging $f$ and $g_{n}$ and using the fact that $L(-f), L\left(-g_{n}\right)$ are finite gives

$$
\left|L(-f)-L\left(-g_{n}\right)\right| \leq L\left|f-g_{n}\right| \text {. }
$$

Replacing $\left(-g_{n}\right)$ with $g_{n}$ and $(-f)$ with $f$ in $(1)$ and then interchanging $f$ and $\boldsymbol{g}_{n}$ gives, as before,

$$
\left|L f-L g_{n}\right| \leq L\left|f-g_{n}\right|
$$

since $L f, L g_{n}$ are finite. From (2) and (3) we have

$$
\begin{aligned}
\mid L(-f) & -(-L f)|\leq| L(-f)-L\left(-g_{n}\right)|+| L\left(-g_{n}\right)-(-L f) \mid \\
& =\left|L(-f)-L\left(-g_{n}\right)\right|+\left|L f-L g_{n}\right| \leq 2 L\left|f-g_{n}\right| \rightarrow 0 \text { as } n \rightarrow \infty .
\end{aligned}
$$

Here we use the fact that $L\left(-g_{n}\right)=M\left(-g_{n}\right)=-M g_{n}=-L g_{n}$. The lemma follows.

4.8 Theorem. If $f \in a_{L}(G)$, then for all $b \in a(G)^{r}$

$$
L_{t} L_{x}|f(x t)-f(t)| b(x) \leq L_{x} L_{t}|f(x t)-f(t)| b(x) .
$$

Proof. By 4.6 the right side of $(1)$ is $M(\Delta, b)$ and, hence, well defined. Also by $4.6, L|f|<\infty$ so by (DL) the left side of (1) is well defined for all $b \in a(G)^{r}$.

We are given $b \in a(G)^{r}$. Take $f_{n} \in a(G)$ such that $\left\|f-f_{n}\right\| \underset{n}{\longrightarrow} 0$. Let $b^{+}, b^{-}$ be the positive and negative parts of $b$ so that $b=b^{+}-b^{-}, 0 \leq^{n} b^{ \pm} \in a(G)^{r}$. We shall show that

$$
L_{t} L\left|f_{t}-f(t)\right| b^{ \pm}=\lim _{n} L_{t} L\left|f_{n t}-f_{n}(t)\right| b^{ \pm}
$$

From (L4), (L5) and the fact that $b^{ \pm} \geq 0$ we have

$$
\begin{aligned}
L\left|f_{t}-f(t)\right| b^{ \pm} & \leq L\left|\left[f_{t}-f_{n t}\right]+\left[f_{n}(t)-f(t)\right]\right| b^{ \pm}+L\left|f_{n t}-f_{n}(t)\right| b^{ \pm} \\
& \leq\left\|b^{ \pm}\right\|_{\infty}\left[\left\|f-f_{n}\right\|+\left|f_{n}(t)-f(t)\right|\right]+L\left|f_{n t}-f_{n}(t)\right| b^{ \pm} .
\end{aligned}
$$

Operating with $L_{t}$ on both sides of (3) gives

$$
L_{t} L\left|f_{t}-f(t)\right| b^{ \pm} \leq 2\left\|b^{ \pm}\right\|_{\infty}\left\|f-f_{n}\right\|+L_{t} L\left|f_{n t}-f_{n}(t)\right| b^{ \pm} .
$$


Observe that $0 \leq L_{t} L\left|f_{t}-f(t)\right| b^{ \pm}<2\left\|b^{ \pm}\right\|_{\infty} L|f|<\infty$ and similarly with $f$ replaced by $f_{n}$. Thus if we interchange $f$ and $f_{n}$ in (4) and combine the two results we get

$$
\left|L_{t} L\right| f_{t}-f(t)\left|b^{ \pm}-L_{t} L\right| f_{n t}-f_{n}(t)\left|b^{ \pm}\right| \leq 2\left\|b^{ \pm}\right\|_{\infty}\left\|f-f_{n}\right\| \underset{n}{\longrightarrow} 0 .
$$

This establishes (2).

Interchanging $f$ and $f_{n}$ in (3), combining the result with (3) and then operating with $L_{t}$ gives

$$
L_{t}|L| f_{t}-f(t)\left|b^{ \pm}-L\right| f_{n t}-f_{n}(t)\left|b^{ \pm}\right| \leq 2\left\|b^{ \pm}\right\|_{\infty}\left\|f-f_{n}\right\| \underset{n}{\longrightarrow} 0 .
$$

That operating with $L_{t}$ is permissible follows from (D2), (D3), the fact that $-L\left|f_{n t}-f_{n}(t)\right| b^{ \pm} \in \alpha(G)$ as a function of $t$, and the fact that $L\left|f_{t}-f(t)\right| b^{ \pm} \in \mathscr{D}$ as a function of $t$ (as was pointed out in the first paragraph of the proof). It follows that

$$
\text { The function } t \rightarrow L\left|f_{t}-f(t)\right| b^{ \pm} \text {is }
$$

$\|$ |l-approximatable by members of $\alpha(G)^{r}$.

From the proof of 4.6 we see that $\left.L\right|_{x} f_{n}-f_{n} \mid \underset{n}{\longrightarrow} \Delta_{f}(x)$ uniformly in $x$. Hence, for arbitrary $\eta>0$,

$$
-\eta<\Delta_{f}(x) b^{ \pm}(x)-\left.L\right|_{x} f_{n}-f_{n} \mid b^{ \pm}(x)<\eta
$$

for all $x \in G$ when $n$ is sufficiently large. Operating on this with $L_{x}$ gives that

$$
\left.L_{x} L\right|_{x} f-f\left|b^{ \pm}(x)=\lim _{n} L_{x} L\right|_{x} f_{n}-f_{n} \mid b^{ \pm}(x) \text {. }
$$

The right sides of (2) and (6) are equal since $f_{n}, b^{ \pm} \in a(G)$. Hence

$$
L_{t} L_{x}|f(x t)-f(t)| b^{ \pm}(x)=L_{x} L_{t}|f(x t)-f(t)| b^{ \pm}(x) .
$$

For each $t, 0 \leq L_{x}|f(x t)-f(t)| b^{+}(x) \leq\left\|b^{+}\right\|_{\infty}[L|f|+|f(t)|]<\infty$. Thus we may apply (L5) to get

$$
L_{t} L_{x}|f(x t)-f(t)| b(x) \leq L_{t}\left[L_{x}|f(x t)-f(t)| b^{+}(x)+L_{x}|f(x t)-f(t)|\left(-b^{-}(x)\right)\right] .
$$

By (5) and $4.70 \leq L_{t} L_{x}|f(x t)-f(t)| b^{+}(x)<\infty$. Thus we may apply (L5) again to get

$$
\begin{aligned}
& L_{t} L_{x}|f(x t)-f(t)| b(x) \\
& \quad \leq L_{t} L_{x}|f(x t)-f(t)| b^{+}(x)+L_{t} L_{x}\left[-|f(x t)-f(t)| b^{-}(x)\right] .
\end{aligned}
$$

Notice that, for fixed $t$,

$$
\left\|\left|f_{t}-f(t)\right| b^{-}-\left|f_{n t}-f(t)\right| b^{-}\right\| \leq\left\|f-f_{n}\right\|\left\|b^{-}\right\|_{\infty} \underset{n}{\longrightarrow} 0 .
$$

Thus for each fixed $t \pm\left|f_{t}-f(t)\right| b-\epsilon \alpha_{L}(G)$. By 4.7, 


$$
L_{x}\left[-|f(x t)-f(t)| b^{-}(x)\right]=-L_{x}|f(x t)-f(t)| b^{-}(x)
$$

for each $t \in G$. As $L|f|<\infty$, (DL) implies that the left and hence the right side of (9) is in $\mathcal{D}$ as a function of $t$. By (5) and 4.7,

$$
L_{t}\left[-L_{x}|f(x t)-f(t)| b^{-}(x)\right]=-L_{t} L_{x}|f(x t)-f(t)| b^{-}(x) .
$$

Combining (7), (8), (9), 10) and the fact that $\Delta \in \alpha(G)$ (by 4.6) gives

$$
\begin{gathered}
L_{t} L_{x}|f(x t)-f(t)| b(x) \leq L_{x} L_{t}|f(x y)-f(t)| b^{+}(x)-L_{x} L_{t}|f(x t)-f(t)| b^{-}(x) \\
=M\left(\Delta_{f} b^{+}\right)-M\left(\Delta_{f} b^{-}\right)=M\left(\Delta_{f} b\right)=L_{x} L_{t}|f(x t)-f(t)| b(x) .
\end{gathered}
$$

This proves the theorem.

4.9 Main Theorem. Let $\alpha(G)$ be a nontrivial module of AP functions on $G$. Let $\mathcal{E}, \mathcal{T}, \mathcal{Q}$ be as in 2.2. Take $f \in \mathcal{E}$. Then $f \in \alpha_{L}(G)$ if and only if

(A) $\Delta_{f}, \Delta_{f}^{r} \in \alpha(G)$,

(B) $L_{t} L_{x}|f(x t)-f(t)| R D_{\rho \rho}^{\lambda}(x) \leq L_{x} L_{t}|f(x t)-f(t)| R D_{\rho \rho}^{\lambda}(x)$ for all $\lambda \in \Lambda\left(\Delta_{f}\right)$ and $1 \leq \rho \leq s_{\lambda}$.

Proof. This follows from 4.5, 4.6 and 4.8.

4.10 Remarks. (1) The requirement in (L1) that $L\left({ }_{x} f\right)=L f$ when $0 \leq f \in \mathcal{E}^{r}$ is used only in the proof of 4.6 and in the proof of $(6)$ in 4.8. The requirement that $L\left(f_{y}\right)=L f$ is used directly in all of the above proofs except 4.7.

(2) Condition (A) is in some cases equivalent to other conditions which appear in AP function theory. For $f \in \mathcal{E}$ let

$$
O_{1}(f)=\left\{{ }_{x} f: x \in G\right\}, \quad O_{2}(f)=\left\{f_{x}: x \in G\right\} .
$$

When $G$ has a topology we say that a symmetric set $S \subset G$ is relatively dense iff there exists a compact set $K \subset G$ such that $K z \cap S \neq \varnothing$ for all $z \in G$ (equivalently: iff there exists compact $F \subset G$ such that $z F \cap S \neq \varnothing$ for all $z \in G$ iff there exists compact $K_{0} \subset G$ such that $G=S K_{0}$, etc.). On the real line this is equivalent to the usual definition of relative density. Notice that $E_{i}(\epsilon, f)$, $L E_{i}(\epsilon, f)$ are symmetric for $\epsilon>0, i=1,2$. Consider the following conditions on a function of $f \in \mathcal{E}$ :

(A0) For every $\epsilon>0$ there exists $w_{1}, \ldots, w_{n}, z_{1}, \ldots, z_{m} \in G$ such that

$$
G=\bigcup_{i=1}^{n} w_{i} L E_{2}(\epsilon, f)=\bigcup_{j=1}^{m} L E_{1}(\epsilon, f) z_{j}
$$

(A1) $\left(O_{i}(f),\|\|\right)$ is totally bounded, $i=1,2$.

(A2) For every $\epsilon>0, L E_{i}(\epsilon, f)$ is relatively dense and open, $j=1,2$. 
4.11 Theorem. Let $f \in$ E. Then

(i) (A) $\Rightarrow$ (A1) $\Leftrightarrow(A 0)$.

(ii) Assume that $(G, \mathcal{I})$ is a topological group and that $a(G) \subset A P(G, \mathcal{J})$. Then $(\mathrm{A}) \Rightarrow(\mathrm{A} 2) \Rightarrow(\mathrm{A} 1) \Leftrightarrow(\mathrm{A} 0)$.

(iii) Assume that $(G, \mathcal{I})$ is a locally compact (or complete metric) topological group, that $a(G)=A P(G, \mathcal{T})$, that every neighborbood of $e$ generates $G$ (or that $\Delta_{f}, \Delta_{f}^{r}$ are finite) and that each $L E_{i}(\epsilon, f)$ is a borel set $(i=1,2)$. Then $(A 0) \Rightarrow(A)$.

Proof. In 2.1 of [5] the equivalency of $(\mathrm{Ai}), 0 \leq i \leq 2$, is shown when $G$ is the real line (usual topology). Therefore we omit details here which resemble those of [5].

(i) That (A1) implies (A0) may be argued as in [5] and the converse is similar. Take $\epsilon>0$. Let us show, for example, that (A) implies that there exists $w_{1}, \cdots, w_{n} \in G$ such that

$$
G=\bigcup_{i=1}^{n} w_{i} L E_{2}(\epsilon, f)
$$

Since $\Delta_{f}^{r} \in \alpha(G),\left(\left\{\left(\Delta_{f}^{r}\right)_{u}: u \in G\right\},\|\|_{\infty}\right)$ is totally bounded. Hence there exists $w_{1}, \cdots, w_{n} \in G$ such that

$$
G=\bigcup_{i=1}^{n} w_{i} E_{2}\left(\epsilon, \Delta_{f}^{r}\right)
$$

By $4.4 E_{2}\left(\epsilon, \Delta_{f}^{r}\right)=L E_{2}(\epsilon, f)$ from which (1) follows.

(ii) Suppose that $(A)$ is true. Then $E_{2}\left(\epsilon, \Delta_{f}^{r}\right)$ is relatively dense for all $\epsilon>0$, because $\Delta_{f}^{r} \in A P(G)$. Since $\Delta_{f}^{r} \in A P(G, \mathcal{J}), \mathcal{J} \supset \mathcal{I}\left(\Delta_{f}^{r}\right)$ by 3.1 . Hence, for

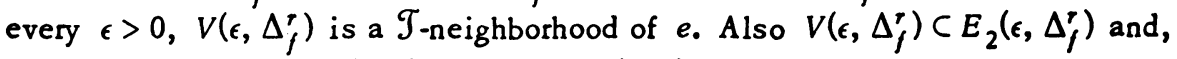
by $4.4, E_{2}\left(\epsilon, \Delta_{f}^{r}\right)=L E_{2}(\epsilon, f)$. Hence $L E_{2}(\epsilon, f)$ is relatively dense and contains

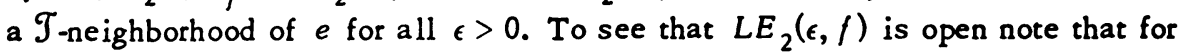
each $x \in L E_{2}(\epsilon, f)$ there exists $\epsilon_{1}<\epsilon$ such that $x \in L E_{2}\left(\epsilon_{1}, f\right)$. Taking $0<\epsilon_{2}<$ $\epsilon-\epsilon_{1}$, let $U \subset L E_{2}\left(\epsilon_{2}, f\right)$ be an open neighborhood of $e$. Then $x \in L E_{2}\left(\epsilon_{1}, f\right) U \subset$ $L E_{2}(\epsilon, f)$. Since $x \in L E_{2}(\epsilon, f)$ is arbitrary, $L E_{2}(\epsilon, f)$ is open. Similarly one shows that $L E_{1}(\epsilon, f)$ is relatively dense and open.

To see that (A2) implies (A0) let us show, for example, that for each $\epsilon>0$ there exists $z_{1}, \ldots, z_{m} \in G$ such that $G=\bigcup_{j=1}^{m} L E_{1}(\epsilon, f) z_{j}$. Take $\epsilon>0$. Take compact $K \subset G$ such that $G=L E_{1}(\epsilon / 2, f) K$. Since $U=L E_{1}(\epsilon / 2, f)$ is an open neighborhood of $e$, there exists $z_{1}, \ldots, z_{m} \in K$ such that $\bigcup_{j=1}^{m} U z_{j} \supset K$. Hence

$$
\bigcup_{j=1}^{m} L E_{1}(\epsilon, f) z_{j} \supset \bigcup_{j=1}^{m} L E_{1}(\epsilon / 2, f) U z_{j} \supset L E_{1}(\epsilon / 2, f) K=G \text {. }
$$

(iii) By Baire's theorem (A0) implies that each $L E_{2}(\epsilon, f)$ is of second 
category. As $L E_{2}(\epsilon, f) \supset\left[L E_{2}(\epsilon / 2, f)\right]^{2}$ and is borel, each $L E_{2}(\epsilon, f)$ contains a neighborhood of $e$. Arguing as in (ii) one deduces that each $L E_{2}(\epsilon, f)$ is open.

Since every neighborhood of $e$ generates $G$,

$$
\dot{G}=\bigcup_{n=1}^{\infty} L E_{2}(1, f)^{n} \subset \bigcup_{n=1}^{\infty} L E_{2}(n, f) \text {. }
$$

It follows that $\Delta_{f}^{r}$ is finite. By $4.4, E_{2}\left(\epsilon, \Delta_{f}^{r}\right)=L E_{2}(\epsilon, f)$ for all $\epsilon>0$. Hence, by (A0), $\Delta_{f}^{r} \in A P(G)$. Since each $E_{2}\left(\epsilon, \Delta_{f}^{r}\right)$ is open, $\mathcal{J} \supset \mathcal{J}\left(\Delta_{f}^{r}\right)$ (see proof of 3.5 ), whence $\Delta_{f}^{r} \in A P(G, \mathcal{J})=\alpha(G)$. Similarly for $\Delta_{f}$. This proves the theorem.

4.12 Remark. We now apply the main theorem to the classical Besicovitch AP functions on $R$. Let $\alpha(R)$ be the set of Bohr AP functions on $R$ and let $L$ be as in 2.3(a). It is customary to denote $L$ by $\bar{M}$. From 4.9, 4.11 and 3.4(2) we have $f \in L_{1, \text { loc }}(R)$ is Besicovitch AP if and only if

(A) $\Delta_{f} \in \alpha(R)$, where $\Delta_{f}(x)=\bar{M}\left|f_{x}-f\right|$, say $\Delta_{f}(x) \sim \Sigma_{k=1}^{\infty} a_{k} e^{i \lambda_{k} x}$; and

(B) $\bar{M}_{t} \bar{M}_{x}|f(x+t)-f(t)| \cos \lambda x \leq \bar{M}_{x} \bar{M}_{t}|f(x+t)-f(t)| \cos \lambda x$ for all $\lambda$ in the additive subgroup of $R$ generated by $\left\{\lambda_{k}\right\}_{k=1}^{\infty} \cup\{0\}$.

(A) may be replaced by any of the equivalent conditions (A0), (A1), or (A2).

References to other known characterizations of $\alpha_{\bar{M}}(R)$ occur in the introduction to [5].(3)

4.13 Theorem. Let $G, \alpha(G), \mathcal{E}, \mathcal{D}, L$ be as in $2.3(\mathrm{~g})$ or $2.3(\mathrm{~h})$. Take $f \in \mathcal{E}$. Then $f \in \alpha_{L}(G)$ if and only if $f$ satisfies (A), (B) and

(M) $t \rightarrow L|f,-f(t)| h$ is $\mu$-measurable for all $b \in \alpha(G)^{r}$.

Proof. Take $f \in a_{L}(G), b \in a(G)^{r}$ and let $\phi(t)=L\left|f_{t}-f(t)\right| b$. Assume first that $\|f\|_{\infty}<\infty$. To prove that $\phi$ is measurable we show that for every $\epsilon>0$ there is a measurable function $\psi$ such that $\|\phi-\psi\|_{\infty}<2 \epsilon$. Take $\epsilon>0$. For each set $E \subset G$ let $\chi_{E}$ denote its indicator. Let $\left\{E_{k}\right\}_{k=1}^{m}$ be a measurable partition of $G$ such that for appropriate $c_{k} \in C$

$$
\left\|f-\sum_{k=1}^{m} c_{k} \chi_{E_{k}}\right\|_{\infty}<\epsilon /\|b\|_{\infty} .
$$

Take $t_{1}, \cdots, t_{n} \in G$ such that for every $t \in G$ there is some $j \in\{1, \cdots, n\}$ satisfying

$$
\left|b\left(x t^{-1}\right)-b\left(x t_{j}\right)\right|<\epsilon / 2\|f\|_{\infty} \text { for all } x \in G \text {. }
$$

Let $\left\{B_{j}\right\}_{1}^{n}$ be a measurable partition of $G$ such that when $t \in B_{j}$ (2) holds.

Let us write $F(t) \approx G(t)$ when $|F(t)-G(t)| \leq \epsilon$. Fix arbitrary $t \in G$. Then

(3) Harold Donnelly has pointed out that the requirement that $f \in L, 10 c(R)$ in the main theorem of $[S]$ may be weakened to measurability. Proofs remain the same. 


$$
\begin{aligned}
\phi(t) & =L_{x}|f(x t)-f(t)| b(x) \\
& \approx L_{x}\left[|f(x t)-f(t)| \sum_{j=1}^{n} b\left(x t t_{j}\right) \chi_{B_{j}}{ }_{j}(t)\right] \text { by }(2) \\
& =\sum_{j=1}^{n} \chi_{B_{j}}(t) L_{x}\left[|f(x t)-f(t)| b\left(x t t_{j}\right)\right] \text { since only one } \chi_{B_{j}} \neq 0 \\
& \approx \sum_{j=1}^{n} \chi_{B_{j}}(t) L_{x}\left[\left|f(x t)-\sum_{k=1}^{m} c_{k} \chi_{E_{k}}(t)\right| b\left(x t t_{j}\right)\right] \text { by (1) } \\
& =\sum_{j, k} \chi_{B_{j} \cap E_{k}}(t) L_{x}\left[\left|f(x t)-c_{k}\right| b\left(x t t_{j}\right)\right] \\
& =\sum_{j, k}\left[L\left|f-c_{k}\right| b_{t}\right] \chi_{B_{j} n E_{k}}(t) \text { by (L1). }
\end{aligned}
$$

The right side is a measurable function within $2 \epsilon$ of $\phi$. Thus $\phi$ is measurable when $\|f\|_{\infty}<\infty$.

If $\|f\|_{\infty}=\infty$, which might happen in $2.3(\mathrm{~h})$, define

$$
f_{n}(x)= \begin{cases}f(x) & \text { if }|f(x)| \leq n, \\ n f(x) /|f(x)| & \text { otherwise. }\end{cases}
$$

It is not difficult to see that $f_{n} \in a_{L}(G)$, in the context of 2.3(h). Define $\phi_{n}(t)=$ $L_{x}\left|f_{n}(x t)-f_{n}(t)\right| b(x)$. Fix $t \in G$ arbitrarily. For large $n$, say $n \geq n_{0}, f_{n}(t)=f(t)$. Now

$$
\begin{aligned}
\left|\phi(t)-\phi_{n}(t)\right| & =|L| f_{t}-f(t)|b-L| f_{n t}-f_{n}(t)|b| \\
& =\left|L\left[\left|f_{t}-f(t)\right| b-\left|f_{n t}-f_{n}(t)\right| b\right]\right|,
\end{aligned}
$$

since $F \in a_{L}(G), H \in a(G)$ implies that $\left|F_{t}-F(t)\right| H \in \alpha_{L}(G)$ for fixed $t$ and $L$ is linear on $a_{L}(G)$. Thus

$$
\begin{aligned}
\left|\phi(t)-\phi_{n}(t)\right| & \leq\|b\|_{\infty}\left|L\left[\left|f_{t}-f(t)\right|-\left|f_{n t}-f(t)\right|\right]\right|, \quad n \geq n_{0}, \\
& \leq\|b\|_{\infty} L\left|f_{t}-f_{n t}\right|=\|b\|_{\infty}\left\|f-f_{n}\right\| \underset{n}{\longrightarrow} 0,
\end{aligned}
$$

by the usual considerations $[4, \mathrm{p} .100]$. By the above $\phi_{n}$ is measurable, hence so is $\phi$. Thus $(M)$ is satisfied and $(A),(B)$ are proven as before.

If $f \in \mathcal{E}$ satisfies $(A),(B)$ and $(M)$, then the argument that $f \in \alpha_{L}(G)$ is as before. This proves the theorem.

4.14 Remarks. (1) Let $G, \alpha(G), E, D, L$ be as in $2.3(\mathrm{~d})$ or $(g) . \alpha_{L}(G)$ is the set of bounded Besicovitch AP functions on $G$ and has been characterized above. The set of Besicovitch AP functions on $G$ (with respect to $L$ ), say $b_{L}(G)$, is defined to be the \|\| -closure of $\alpha(G)$ in $L_{1, \text { loc }}(G)$. Take $f \in L_{1, \text { loc }}(G)$. Define 


$$
f_{n}(x)= \begin{cases}f(x) & \text { if }|f(x)| \leq n, \\ n f(x) /|f(x)| & \text { otherwise, } n=1,2, \ldots .\end{cases}
$$

Then $f \in B_{L}(G)$ iff each $f_{n}$ satisfies (A), (B), (M) and $\left\|f_{n}-f\right\| \underset{n}{\longrightarrow} 0$. (M) may be omitted in the case of $2.3(\mathrm{~d})$.

$\left(B_{L}(G),\|\|\right)$ may not be complete even though the sets $\left\{V_{d}\right\}_{d \in D}$ defining $L$ satisfy

$$
\lim _{d \in D} \frac{\mu\left(x V_{d} \Delta V_{d}\right)}{\mu\left(V_{d}\right)}=0 \text { for all } x \in G .
$$

The example in $\S_{5}$ of [8] illustrates this. (4) Even when $B_{L}(G)$ is not complete its members have Fourier series which are unique and may be "summed" to the original function. When $B_{L}(G)$ is complete, it is a copy of $L_{1}(\bar{G})$, where $\bar{G}$ is the Bohr compactification of $G$ (see $[8,(\varsigma) \S \S 3,41$; completeness is not assumed in \$3).

(2) The Weyl AP functions on an LC group (2.3(h)) are characterized in 4.13. Another characterization along more classical lines is in [7]. The classical characterization on $R$ may be found in [4]. Different characterizations of Weyl AP functions on $R$ occur in [9] and [12] (cf. remarks on pp. 23, 25 of [12]).

(3) There are some interesting Besicovitch spaces for which 4.9 does not appear applicable. For example, suppose $(G, \mathfrak{I})$ is a noncommutative $\sigma$-compact LC group satisfying at least one of the following:

(i) $G$ is amenable,

(ii) $G$ is first countable and nondiscrete,

(iii) $G$ is free and discrete.

Then there is a sequence of open bounded subsets $\left\{V_{n}\right\}_{1}^{\infty}$, increasing to $G$ such that

$$
\lim _{n \rightarrow \infty} \frac{1}{\mu\left(V_{n}\right)} \int_{V_{n}} f d \mu=M f
$$

for all $f \in A P(G, \mathcal{J})$ (cf., $[11,3.2] ;[6]$ ). If we define $L$ on $L_{1,10 c}(G)^{r}$ (or even on its bounded members) by

$$
L f=\varlimsup_{n \rightarrow \infty} \frac{1}{\mu\left(V_{n}\right)} \int_{V_{n}} f d \mu,
$$

(4) The sets $V_{d}$ defined there are not open. They may be assumed open by taking the $W(n, a)$ 's to be open and using approximation considerations in case 1 of $\$ 5$.

(5) In $\oint_{3}$ of $[8] \sigma(G)$ should be defined as $[h]$, where $h$ is any AP functions on $G$ whose nonzero Fourier matrices are $\left\{D^{\lambda}: \lambda \in \Sigma\right\}$. This is not equivalent to the definition given. $A$ similar modification of the definition of $\sigma^{\prime}(G)$ in the proof of 3.1 should be made. 
(L1) may not hold. It is not apparent that one can get around this difficulty in the fashion used in $2.3(\mathrm{~g}),(\mathrm{h})$. The closure of $A P(G, \mathfrak{J})$ in $L_{1, l o c}(G)$ by means of $L$ is complete. The resulting Besicovitch space is a copy of $L_{1}(\bar{G})$, where $\bar{G}$ is the Bohr compactification of $G[8,2.4$ and $\$ 4]$.

5. Weakly AP functions are contained in the generalized AP functions. Let $(G, \mathcal{I})$ be an LC group. Let $C B(G, \mathcal{I})$ be the continuous bounded complex-valued functions on $G$ and let $W A P(G, \mathcal{I})$ be the weakly AP functions on $G: f \in W A P(G, \mathcal{T})$ iff $f \in C B(G, \mathfrak{N})$ and $O(f)=\{x f: x \in G\}$ is relatively compact in the weak topology of $\left(C B(G, \mathfrak{T}),\|\|_{\infty}\right)$. For $f \in W A P(G, \mathfrak{T}), M f$ denotes its mean value. We shall use such basic facts about $W A P(G, \mathfrak{T})$ as may be found in [3].

If $(G, \mathfrak{I})$ is amenable, there is a directed set $\left(V_{d}, d \in D, \geq\right)$ of $\mu$-positive bounded (= having compact closure) subsets of $G$ such that

$$
\lim _{d \in D} \frac{\mu\left(x V_{d} \Delta V_{d}\right)}{\mu\left(V_{d}\right)}=0 \text { for all } x \in G .
$$

In addition one may require that

(b) $d_{1} \leq d_{2}$ implies $V_{d_{1}} \subset V_{d_{2}}$;

(c) $\bigcup_{d \in D} V_{d}=G$; and

(d) each $V_{d}$ is open and bounded.

See [11] and [15, p. 43]. There the $V_{d}$ 's are compact but regularity of $\mu$ allows one to substitute slightly larger open sets in the conditions $\left(A_{10 c}\right)$ of [11].

5.1 Theorem. Let $(G, \mathcal{T})$ be an LC group.

(i) There is a net $\left(V_{d}, d \in D, \geq\right)$ of subsets of $G$ satisfying (b), (c), (d) and

$$
\lim _{d \in D} \frac{1}{\mu\left(V_{d}\right)} \int_{V_{d}} f d \mu=M f \text { for all } f \in W A P(G, \mathfrak{T}) \text {. }
$$

(ii) Every net $\left(V_{d}, d \in D, \geq\right)$ of $\mu$-positive bounded sets satisfying (a1) also satisfies (a2).

(iii) If $(G, \mathfrak{T})$ is abelian (bence amenable) and $\left(V_{d}, d \in D, \geq\right)$ is a net of $\mu$-positive bounded sets of $G$ satisfying (a1), then

(a3) for every $f \in W A P(G, \mathcal{J})$

$$
\lim _{d \in D} \frac{1}{\mu\left(V_{d}\right)} \int_{V_{d}} f_{x} d \mu=M F \quad \text { uniformly in } x \in G .
$$

5.2 Remark. (i) answers a question raised by Greenleaf in [15, p. 43]. There it is pointed out that (ii) follows from the argument of $[17,18.10]$. I do not know if (a2) is true uniformly with respect to (one or two-sided) translations of $\%$. It is if $f \in A P(G, \mathfrak{I})$.

Proof of 5.1. We need only prove (i), (iii). We use the notation of $[3$, see especially p. 146]. Let $\Omega G$ be the weak compactification of $G$ and $\omega_{G}: G \rightarrow \Omega G$ 
canonical. $M(\Omega G)$ denotes the minimal ideal of $\Omega G$. Recall that $\omega_{G}$ imbeds $G$ isomorphically and homeomorphically onto an open dense subset of $\Omega G$. We often identify $G$ with $\omega_{G}(G)$. We may as well assume $G$ is noncompact.

To prove (i) we establish several propositions $[A],[B],[C]$ :

[A] If $K \subset G$ is compact, $G \sim K$ is dense in $\Omega G \sim K$.

For otherwise there exists $x \in \Omega G \sim K$ such that $x \notin(G \sim K)^{-\Omega G}$. But then $x \in \bar{K}^{\Omega G}=\bar{K}^{G}=K$, a contradiction.

[B] Let $f_{1}, \ldots, f_{n} \in W A P(G, I)$ and let $U$ be a bounded open neighborhood of $e$ in $G$. Take $N, \epsilon>0$. There exists distinct $a_{1}, \ldots, a_{t} \in G$ such that

$$
\left|\frac{1}{t} \sum_{i=1}^{t} f_{k}\left(a_{i}\right)-M f_{k}\right|<\epsilon, \quad 1 \leq k \leq n, t \geq N \text {, and } a_{i} U \cap a_{j} U=\varnothing \text { if } i \neq j \text {. }
$$

To prove [B] notice that each $f_{k} / M(\Omega G)$ is AP since it is continuous on $M(\Omega, G)$, a compact group. We apply IV.1.1(f) of [3]. If $M(\Omega G)$ is finite, say with cardinality $p$, let $b_{1}, \ldots, b_{t}$ be a listing of the elements of $M(\Omega G)$ such that each element occurs $q$ times in the list and $p q \geq N$. If $M(\Omega G)$ is infinite, apply 3.3 of [G] to obtain $b_{1}, \ldots, b_{t} \in M(\Omega G)$ such that $t \geq N$ and

$$
\left|\frac{1}{t} \sum_{i=1}^{t} f_{k}\left(b_{i}\right)-M f_{k}\right|<\epsilon / 2, \quad 1 \leq \dot{k} \leq n \text {. }
$$

By continuity of $f_{k}$ on $\Omega G$ and density of $G$ in $\Omega G$ there exists $a_{1} \in G$ such that $\left|f_{k}\left(b_{1}\right)-f_{k}\left(a_{1}\right)\right|<\epsilon / 2,1 \leq k \leq n$. By $[\mathrm{A}]$ and continuity of each $f_{k}$, there exists $a_{2} \in G$ such that $a_{2} U \cap a_{1} U=\varnothing$ (take $a_{2} \in G \sim a_{1}\left(U U^{-1}\right)-$ ) and $\left|f_{k}\left(b_{2}\right)-f_{k}\left(a_{2}\right)\right|<\epsilon / 2,1 \leq k \leq n$. (Recall that $M(\Omega G)$ and $\omega_{G}(G)$ are disjoint.) Continuing inductively one gets $a_{1}, \ldots, a_{t} \in G$ which evidently satisfy the required conditions.

[C] Let $f_{1}, \cdots, f_{n} \in W A P(G, \mathfrak{T})$ and take $M, \epsilon>0$. There exists a bounded open set $B \subset G$ such that $\mu(B) \geq M$ and

$$
\left|M f_{k}-\frac{1}{\mu(B)} \int_{B} f_{k} d \mu\right|<2 \epsilon, \quad 1 \leq k \leq n \text {. }
$$

To prove this use uniform continuity of the $f_{k}$ to get a bounded open neighborhood $U$ of $e$ in $G$ such that $\left|f_{k}(x u)-f_{k}(x)\right|<\epsilon, 1 \leq k \leq n$, whenever $x \in G$, $u \in U$. Take $N$ such that $N \mu(U) \geq M$ and let $a_{1}, \ldots, a_{t} \in G$ be as in [B]. It is not difficult to see that $B=\bigcup_{i=1}^{t} a_{i} U$ has the required properties.

To finish the proof of (i), one applies the arguments of 3.4 in [6], replacing $\alpha(G)$ and 3.3 in [G] by $\operatorname{WAP}(G, I)$ and [C] above.

To prove (iii) consider $G$ as acting on $\left(W A P(G, \mathfrak{J}),\|\|_{\infty}\right)$ via left translation. The elements of $W A P(G, \mathcal{J})$ are ergodic in the sense of Eberlein (Definition 3.1 of [10]). Thus it suffices to show that the transformations $T_{d}$ defined on $\operatorname{WAP}(G, \mathfrak{I})$ by 


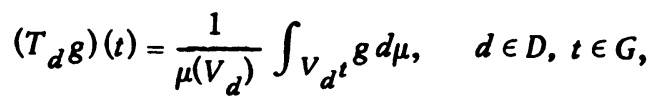

are a system of almost invariant integrals (in the sense of Eberlein's Definition 2.1 of [10]). It is clear that each $T_{d}: W A P(G, \mathfrak{I}) \rightarrow C B(G, \mathfrak{I})$ is linear and that $\left\{T_{d}\right\}_{d \in D}$ is equicontinuous. That $T_{d} g \in$ [convex hull of $\left.O(g)\right]^{-} C W A P(G, \mathfrak{I})$ for each $g \in W A P(G, \mathfrak{I})$ is not difficult to show from the uniform continuity of $g$ (one approximates $(1 / \mu(V)) \int_{V} x^{g} d \mu$ by $\sum_{i=1}^{n}\left(\left(\mu\left(V_{i}\right) / \mu(V)\right)_{g}\left(x t_{i}\right)\right)$. Finally we must show that for each $g \in W A P(G, \mathfrak{T}), x \in G$,

Now

$$
\lim _{d \in D}\left\|_{x}\left(T_{d} g\right)-T_{d} g\right\|_{\infty}=\lim _{d \in D}\left\|T_{d}\left({ }_{x} g\right)-T_{d} g\right\|_{\infty}=0
$$

$$
x_{x}\left(T_{d} g\right)(t)=\left[T_{d}\left({ }_{x} g\right)\right](t)=\frac{1}{\mu\left(V_{d}\right)} \int_{V_{d} t x} g d \mu,
$$

since $G$ is abelian. Thus

$$
\left|{ }_{x}\left(T_{d} g\right)(t)-\left(T_{d} g\right)(t)\right|=\left|\left[T_{d}\left({ }_{x} g\right)\right](t)-\left(T_{d} g\right)(t)\right| \leq \frac{\|g\|_{\infty} \mu\left(x V_{d} \Delta V_{d}\right)}{\mu\left(V_{d}\right)} \underset{d \in D}{\longrightarrow} 0
$$

uniformly in $t \in G$. This proves the theorem.

5.3 Remark. We may write $W A P(G, \mathfrak{T})=A P(G, \mathfrak{I}) \oplus W A P(G, \mathfrak{T})_{0}$ where $W A P\left(G, I_{)_{0}}=\left\{f \in W A P(G, \mathfrak{T}): M|f|^{2}=0\right\}\right.$. Since, for each $f \in W A P(G, \mathfrak{I}), M f$ may be uniformly approximated by finite convex sums of the form

$$
(S f)(x)=\sum_{r} a_{r} f\left(x a_{r}\right) \quad\left(a_{r} \in G ; a_{r}>0, \sum a_{r}=1\right)
$$

and since $\|f\|_{\infty} S|f| \geq S|f|^{2} \geq(S|f|)^{2}$, we may write $W A P\left(G, \mathfrak{N}_{0}=\{f \in W A P(G, \mathfrak{I})\right.$ : $M|f|=0\}$. Let $\Phi=\left(V_{d}, d \in D, \geq\right)$ be a net $\mu$-positive bounded set satisfying condition (a2) of 5.1. Define $W(G, \Phi), B(G, \Phi)$ to be corresponding spaces of Weyl and Besicovitch AP functions on $G$, obtained by closing $A P(G, \mathfrak{T})$ in $L_{1, \text { loc }}(G)$ with the seminorm of $2.3(\mathrm{~h}), 2.3(\mathrm{~g})$ respectively. By allowing (a2) rather than (al), we allow $B(G, \Phi)$ to be more general than in $2.3(\mathrm{~g})$. The characterization Theorems 4.9, 4.13 and 4.14(1) may not apply to $B(G, \Phi)$ unless $G$ is abelian and (al) holds. The elements of $W(G, \Phi)$ and $B(G, \Phi)$ have mean values given by

$$
M_{\phi} f=\lim _{d \in D} \frac{1}{\mu\left(V_{d}\right)} \int_{v_{d}} f d \mu \text {. }
$$

Define $W(G, \Phi)_{0}=\left\{f \in W(G, \Phi): M_{\Phi}|f|=0\right\}$ and similarly for $B(G, \Phi)_{0}$. For $f \in W(G, \Phi)$, the limit in $(\dagger)$ is uniform with respect to translation of $f$ (see, for example, the "proposition"' of [9]). Hence we may write $W(G, \Phi)_{0}=$ $\left\{f \in L_{1, \text { loc }}(G):\|f\|=0\right\}$. Here \|\| is the seminorm defined in 2.2 from $L$ as defined in 2.3(h). Similarly, $B(G, \Phi)_{0}=\left\{f \in L_{1, \text { loc }}(G):\|f\|=0\right\}$ for \|\| as in $2.3(\mathrm{~g})$. 
The following extends Eberlein's theorem [10, \#16] concerning the Weyl almost periodicity of weakly AP functions on $R$. It follows from 5.1 and the above discussion.

5.4 Corollary to 5.1. Let $(G, \mathfrak{J})$ be an $L C$ group and let $\Phi=\left(V_{d}, d \in D_{0} \geq\right)$ be as above. Assume (a2) of 5.1 bolds. Then WAP(G, $\mathfrak{I}_{0} \subset B(G, \Phi)_{j}$ and bence $\operatorname{WAP}(G, \mathcal{I}) \subset B(G, \Phi)$. If (a3) bolds, then $W A P\left(G, \mathfrak{I}_{0} \subset W(G, \Phi)_{0}\right.$ and bence $W A P(G, \mathcal{J}) \subset W(G, \Phi)$.

5.5 Remarks. (1) Suppose $(G, \mathfrak{I})$ is of the form $R^{a} \times Z^{b} \times F$, where $F$ is compact and $Z=\{0, \pm 1, \cdots\}$ (see $2.3(\mathrm{c}))$. Let $V_{n}=(-n, n)^{a+b} \times F, n=1,2, \cdots$, in 5.1. In this case (a3) of 5.1 is satisfied even when $G$ is not abelian. Thus in this case $W A P(G, \mathfrak{I}) \subset W(G, \Phi)$.

(2) One cannot expect $W A P(G, \mathfrak{T})_{0}=W(G, \Phi)_{0} \cap C B(G, \mathfrak{T})$ even when $G$ is abelian. For example, suppose $G=\{0, \pm 1, \ldots\}, \mathcal{T}$ is discrete and $\Phi=(\{1, \cdots, n\}$, $n=1,2, \cdots, \geq)$. Define $E=\left\{3^{j}-2^{i}: j=1,2, \cdots ; 1 \leq i \leq j\right\}$ and let $\chi_{E}$ be the indicator of $E$. Then $\chi_{E} \in W(G, \Phi)_{0} \cap C B(G, \mathfrak{J})$. But

$$
\lim _{i} \lim _{j} \chi_{E}\left(3^{j}-2^{i}\right)=1, \quad \lim _{i} \lim _{j} \chi_{E}\left(3^{j}-2^{i}\right)=0
$$

so $\chi_{E} \notin W A P(G, \mathcal{I})[16$, Proposition 7$]$.

\section{REFERENCES}

1. H. Anzai and S. Kakutani, Bohr compactifications of a locally compact abelian group. I, Proc. Imp. Acad. Tokyo 19 (1943), 476-480. MR 7, 374.

2. J. Berglund, On extending almost periodic functions, Pacific J. Math. 33 (1970), 281-289.

3. J. Berglund and K. Hofmann, Compact semitopological semigroups and weakly almost periodic functions, Lecture Notes in Math., no. 42, Springer-Verlag, Berlin and New York, 1967. MR 36 \#6531.

4. A. Besicovitch, Almost periodic functions, Dover, New York, 1955. MR 16, 817.

5. A. Dabboucy and H. Davis, $A$ new characterization of Besicovitch almost periodic functions, Math. Scand. 28 (1971), 341-354.

6. H. Davis, On the mean value of Haar measurable almost periodic functions, Duke Math. J. 34 (1967), 201-214. MR 35 \#2995.

7. - Stepanoff and Weyl AP functions on locally cbmpact groups, Duke Math. J. 34 (1967), 535-548. MR 35 \#6779.

8. - On completing the Von Neumann almost periodic functions, Duke Math. J. 35 (1968), 199-216. MR 36 \#5614. 51-61.

9. - On a formula for Haar measure in compact groups, Math. Scand. 30 (1972),

10. W. Eberlein, Abstract ergodic theorems and weak almost periodic functions, Trans. Amer. Math. Soc. 67 (1949), 217-240. MR 12, 112.

11. W. Emerson and F. Greenleaf, Covering properties and F plner conditions for locally compact groups, Math. Z. 102 (1967), 370-384. MR 36 \#3912. 
12. E. Folner, On the structure of generalized almost periodic functions, Danske Vid. Selsk. Mat.-Fys. Medd. 21 (1945), no. 11, 1-30. MR 7, 519.

13. - W-almost-periodic functions on arbitrary groups, Mat. Tidsskr. B. 1946, 153-162. (Danish) MR 8, 14.

14. - Besicovitch almost periodic functions in arbitrary groups, Math. Scand. 5 (1957), 47-53. MR 19, 958.

15. F. Greenleaf, Invariant means on topological groups and their applications, Van Nostrand Math. Studies, no. 16, Van Nostrand Reinhold, New York, 1969. MR 40 \#4776.

16. A. Grothendieck, Critères de compacité dans les espaces fonctionnels généreaux, Amer. J. Math. 74 (1952), 168-186. MR 13, 857.

17. E. Hewitt and K. Ross, Abstract harmonic analysis. Vol. I: Structure of topological groups. Integration theory, group representations, Die Grundlehren der math. Wissenschaften, Band 115, Academic Press, New York; Springer-Verlag, Berlin, 1963. MR 28 \#158.

18. E. van Kampen, Almost periodic functions and compact groups, Ann. of Math. 37 (1936), 78-91.

19. W. Maak, Fastperiodische Funktionen, Die Grundlehren der math. Wissenschaften, Band 61, Springer-Verlag, Berlin, 1950. MR 13, 29.

APPLIED MATHEMATICS DEPARTMENT, BROOKHAVEN NATIONAL LABORATORY, UPTON, NEW YORK 11973

Current address : Department of Computer Science, State University of New York at Stony Brook, Stony Brook, New York 11790 